\title{
INFLUENCIA DE LAS ESTRATEGIAS DE APRENDIZAJE Y LA REFLEXIÓN ACTIVA SOBRE EI RENDIMIENTO ESCOLAR DE LOS ALUMNOS DE QUINTO AÑO DE SECUNDARIA DE LA CIUDAD DE LIMA
}

\author{
INFLUENCES OF THE STRATEGIES OF LEARNING AND THE ACTIVE \\ REFLECTION ON THE SCHOLASTIC YIELD OF THE STUDENTS OF FIFTH \\ YEAR OF SECONDARY OF THE CITY OF LIMA
}

\author{
LUIS MIGUEL ESCURRA MAYAUTE ${ }^{1}$, ANA DELGADO VÁSQUEZ, AMPARO \\ SOTIL BRIOSO, JUAN PEQUEÑA CONSTANTINO, MARÍA ROSARIO \\ QUESADA MURILLO, GERARDO RIVAS CASTRO, ROLANDO SOLIS \\ NARRO, JULIO SANTOS ISLAS \\ Facultad de Psicología, UNMSM
}

\section{Resumen}

OBJETIVO: Estudiar la influencia de las estrategias de aprendizaje y la reflexión activa sobre el rendimiento escolar de los alumnos de quinto año de secundaria de la ciudad de Lima. DISEÑO: Estudio correlacional multivariado, realizado en alumnos de secundaria de Lima y Callao en el año 2003. MATERIAL Y MÉTODOS: Muestreo por conglomerados en dos etapas, con probabilidades proporcionales al tamaño; la muestra para el estudio estuvo conformado por 1200 adolescentes. Se utilizó la escala ACRA y se construyó una escala de Reflexión Activa. El análisis estadístico se desarrolló en tres etapas, psicométrico para evaluar la validez y confiabilidad del ACRA y la Escala de Reflexión Activa; descriptivo de las variables estudiadas, inferencial multivariado (modelo de ecuaciones estructurales) y bivariado para contrastar las hipótesis planteadas. RESULTADOS: La media de la edad de los adolescentes encuestados fue de 16,1 $\pm 0,86$ años, el $52.0 \%$ fueron mujeres y el $48.0 \%$ fueron varones. Los alumnos pertenecientes a colegios estatales fueron el $53.8 \%$, los de colegios particulares el $46.2 \%$, los alumnos nacidos en Lima fueron el 61.1\%. CONCLUSIONES: Los hallazgos indican que las escalas ACRA y de Reflexión Activa presentan validez y confiabilidad. Los puntajes de los alumnos en las áreas de la escala ACRA, la escala de reflexión adiva y el rendimiento académico, presentan distribuciones que se aproximan a la curva normal. Existen diferencias significativas en las estrategias de aprendizaje y la reflexión adiva por tipo de gestión del colegio. Existen diferencias significativas en las Estrategias de aprendizaje y la Reflexión Adiva por género y las estrategias de aprendizaje y la reflexión activa han demostrado que influyen de manera positiva sobre el rendimiento Escolar.

Palabras Claves: Estrategias de aprendizaje; reflexión activa; rendimiento escolar; alumnos de secundaria; modelo de ecuaciones estructurales

\begin{abstract}
OBJECTIVE: To determine the influence of learning strategies and the active reflection on the academic performance of senior high school students of the city of Lima. DESIGN: Multivariate correlational study carried on Lima and Callao high school students of the 2003 class. INSTRUMENTS AND METHODS: A two stages conglomerate sampling afixed to size was used, resulting 1200 adolescents as participants. The ACRA scale was used and an Active Reflection scale was elaborated. The statistics analysis was done in three stages: A psychometric analysis to evaluate validity and reliability of ACRA's and Active Reflection scales, a descriptive analysis of the studied variables, and a multivariate (structural equation modeling) and bivariate analysis to test the hiphotesis. RESUL TS: The adolescents mean age was 16.1 year (with.土. 0.86 error aproximation). The $52 \%$ were female and the $48 \%$ were male. The students from public schools were the $53.8 \%$ and the ones from private schools were the 46.2\%. Those from Lima were $61.1 \%$. CONCLUSIONS: The results indicated that both instruments, ACRA's and Active Reflection scales are valid and reliable. The students scores in both ACRA's and Active
\end{abstract}


Reflection scales, and academic performance are normally distributed. There are significative differences in learning strategies and active reflection when each variable is analized considering school management. There are significative differences both in learning strategies and active reflection considering gender, also learning strategies and active reflection afects positively academic performance.

Keywords: Learning strategies, active reflection, academic performance, high school students, structural equation modeling.

${ }^{1}$ Email: lescurram@unmsm.edupe 


\section{INTRODUCCIÓN}

Las estrategias de aprendizaje en la educación actual, son muy importantes en la medida que incluyen aquellos recursos cognitivos que utiliza el estudiante cuando se enfrenta al aprendizaje, así como aquellos elementos directamente vinculados tanto con la disposición y motivación del estudiante como en las actividades de planificación, dirección y control que el sujeto pone en marcha cuando se enfrenta al aprendizaje. Por lo tanto, al hablar de estrategias no solo se alude a como aprender, sino que también se toman en cuenta las razones, intenciones y motivos que guían el aprendizaje junto con las actividades de planificación, dirección y control de todo este proceso.

En términos conceptuales las estrategias de aprendizaje pueden definirse siguiendo a Klauer (1988) ${ }^{1}$ como secuencias de acción dirigidas a la obtención de metas de aprendizaje, por lo que representan complejas operaciones cognitivas que son antepuestas a los procedimientos específicos de la tarea.

Para Weinstein y Mayer $(1986)^{2}$, las estrategias de aprendizaje pueden ser definidas como conductas y pensamientos que un aprendiz utiliza durante el aprendizaje con la intención de influir en su proceso de codificación.

Para Monereo $(2000)^{3}$, las estrategias de aprendizaje son procesos de toma de decisiones (conscientes e intencionales) en los cuales el alumno elige y recupera, de manera coordinada, los conocimientos que necesita para cumplimentar una determinada demanda $\mathrm{u}$ objetivo, dependiendo de las características de la situación educativa en que se produce la acción.

Según Genovard y Gotzens $(1996)^{4}$, las estrategias de aprendizaje pueden .definirse como los comportamientos que el estudiante despliega durante su proceso de aprendizaje y que, supuestamente, influyen en su proceso de codificación de la información que debe aprender.

A partir de estas definiciones, se puede afirmar que existen coincidencias entre los autores más representativos en este campo en resaltar algunos elementos importantes del concepto de estrategias de aprendizaje. Por una parte, las estrategias implican una secuencia de actividades, operaciones o planes dirigidos a la consecución de metas de aprendizaje; y por otra tienen un carácter consciente e intencional en el que están implicados procesos de toma de decisiones por parte del alumno ajustados al objetivo o meta que pretende conseguir.

De acuerdo con Beltrán (1996) ${ }^{5}$, éstas definiciones ponen en relieve dos aspectos importantes: En primer lugar, se trata de actividades u operaciones mentales que realiza el estudiante para mejorar el aprendizaje. En segundo lugar, las estrategias tienen un carácter intencional o propositivo e implican, por tanto, un plan de acción.

Las estrategias de aprendizaje constituyen actividades conscientes e intencionales que guían las acciones a seguir para alcanzar determinadas metas de aprendizaje. Son actividades potencialmente conscientes y controlables, que están bajo el control del estudiante, es decir, a pesar de que ciertas rutinas pueden ser aprendidas hasta el punto de automatizarse, las estrategias son generalmente deliberadas, planificadas y conscientemente comprometidas en actividades. Dicho en otros términos, las estrategias de aprendizaje son procedimientos que se aplican de un modo intencional y deliberado a una tarea y que no pueden reducirse a rutinas automatizadas, es decir, son más que simples secuencias o aglomeraciones de habilidades. Como afirma Beltrán (1996) ${ }^{5}$, las estrategias tienen un carácter intencional; implican, por tanto, un plan de acción, frente a la técnica, que es marcada mente mecánica y rutinaria.

En términos generales, las estrategias de aprendizaje son representadas mentalmente como planes de acción. Borkowsky, Carr \& Pressley (1987) ${ }^{6}$, han demostrado que la aparente espontaneidad en el uso que una persona hace de las estrategias de aprendizaje es, en realidad, el resultado de una interacción compleja que incluye el conocimiento que tiene 
de las estrategias, el conocimiento de las estrategias de coordinación de alto nivel y sus creencias motivacionales.

Desde un punto de vista evolutivo, con la adquisición de estrategias de aprendizaje y de pensamiento, específicas para un contexto en diferentes campos de conocimiento, se edifican al mismo tiempo potenciales estrategias más generales. El conocimiento acerca de la estrategia es en un comienzo, poco estructurado internamente. En el transcurso del aprendizaje escolar y con el ensanchamiento de la base de conocimiento disponible se debería diferenciar el repertorio de estrategias, tanto que la implementación de estrategias de aprendizaje puede llegar a ser un proceso de adaptación crecientemente flexible. Los rasgos característicos más destacados de las estrategias de aprendizaje son las siguientes:

a. Su aplicación no es automática, sino controlada. Precisan planificación y control de la ejecución y están relacionadas con la metacognición o conocimiento sobre los propios procesos mentales.

b. Implican un uso selectivo de los propios recursos y capacidades disponibles. Para que un estudiante pueda poner en, marcha una estrategia debe disponer de recursos alternativos, entre los que decide utilizar, en función de las demandas de la tarea, aquéllos que él cree más adecuados.

c. Las estrategias están constituidas de otros elementos más simples, que son las técnicas o tácticas de aprendizaje y las destrezas o habilidades. De hecho, el uso eficaz de una estrategia depende en buena medida de las técnicas que la componen. En todo caso, el dominio de las estrategias de aprendizaje requiere, además de destreza en el dominio de ciertas técnicas, una reflexión profunda sobre el modo de utilizarlas o, en otras palabras, un uso reflexivo - y no sólo mecánico o automático - de las mismas.

Weinstein y Mayer (1986) ${ }^{2}$ distinguen tres grandes grupos de estrategias:

1. Las estrategias cognitivas, aluden a la integración del nuevo material con el conocimiento previo. En este sentido, serían un conjunto de estrategias que se utilizan para aprender, codificar, comprender y recordar la información al servicio de unas determinadas metas de aprendizaje. Dentro de este grupo se distinguen a su vez tres clases de estrategias: La estrategia de repetición, consiste en pronunciar, nombrar o decir de forma repetida los estímulos presentados dentro de una tarea de aprendizaje. Se trataría, por tanto, de un mecanismo de la memoria a corto plazo y a la vez, transferidos a la memoria a largo plazo. Por otro lado, mientras que la estrategia de elaboración trata de integrar los materiales informativos relacionando la nueva información con la ya almacenada en la memoria. La estrategia de organización intenta combinar los elementos informativos seleccionados en un todo coherente y significativo. Además, dentro de esta categoría de estrategias cognitivas también estarían las estrategias de selección o esencialización, cuya función principal es la de seleccionar aquella información más relevante con la finalidad de facilitar su procesamiento.

2. Las estrategias metacognitivas hacen referencia a la planificación, control y evaluación por parte de los estudiantes de su propia cognición. Son un conjunto de estrategias que permiten el conocimiento de los procesos mentales, así como el control y regulación de los mismos con el objetivo de lograr determinadas metas de aprendizaje. Según Kirby $(1997)^{7}$, este tipo de estrategias sería macroestrategias, ya que son más complejas que las anteriores, presentan un elevado grado de transferencia, son menos susceptibles de ser enseñadas, y están estrechamente relacionadas con el conocimiento metacognitivo.

El conocimiento metacognitivo requiere conciencia y conocimiento de variables de la persona, de la tarea y de la estrategia. En relación con las variables personales está la conciencia y conocimiento que tiene el sujeto de sí mismo y de sus capacidades y limitaciones cognitivas; aspecto que se va formando a partir de las percepciones y comprensiones que desarrollamos nosotros mismos, en tanto sujetos que aprenden y piensan. Las variables de la tarea se refieren a la reflexión sobre el tipo de problema que se 
va a tratar de resolver. Significa, por tanto, averiguar el objetivo de la tarea. En este sentido, puede entenderse la conciencia (conocimiento) metacognitiva como un proceso de utilización de pensamiento reflexivo para desarrollar la conciencia y conocimiento sobre uno mismo, la tarea, y las estrategias en un contexto determinado.

Por consiguiente, una buena base de conocimientos de las características y demandas de la tarea, de las capacidades, intereses y actitudes personales, y de las estrategias necesarias para completar la tarea, son requisitos básicos de la conciencia y conocimientos metacognitivo; a lo que debemos de añadir la regulación y control que el propio sujeto debe ejercer sobre todo lo anterior. Para Kurtz-Costes y Schneider (1994) ${ }^{8}$, la metacognición regula de dos formas el uso eficaz de las estrategias: en primer lugar, para que un individuo pueda poner en práctica una estrategia, antes debe tener conocimiento de estrategias específicas y saber cómo, cuándo y por qué debe usarlas. Así, por ejemplo, debe conocer las técnicas de repaso, subrayado, resumen, etc.; y saber cuándo conviene utilizarlas. En segundo lugar, mediante su función autorreguladora, la metacognición hace posible observar la eficacia de las estrategias elegidas y cambiarlas según las demandas de la tarea. Las estrategias metacognitivas equivalen a lo que Weinstein y Mayer (1986) 2 denominan como estrategias de control de la comprensión. Estas estrategias están formadas por procedimientos de autorregulación que hacen posible el acceso consciente a las habilidades cognitivas empleadas para procesar la información. Para estos autores, un estudiante que emplea estrategias de control es también un estudiante metacognitivo, ya que es capaz de regular el propio pensamiento en el proceso de aprendizaje.

3. Las estrategias de manejo de recursos, son una serie de estrategias de apoyo que incluyen diferentes tipos de recursos que contribuyen que la resolución de la tarea se lleve a buen término. Tienen como finalidad sensibilizar al estudiante con lo que va a aprender; y esta sensibilización hacia el aprendizaje integra tres ámbitos: la motivación, las actitudes y el afecto. Este tipo de estrategias coinciden con lo que Weinstein y Mayer (1986) ${ }^{2}$ llaman estrategias afectivas y otros autores denominan estrategias de apoyo, e incluyen aspectos claves que condicionan el aprendizaje como son el control del tiempo, la organización del ambiente de estudio, el manejo y control del esfuerzo, etc. Este tipo de estrategias, en lugar de enfocarse directamente sobre el aprendizaje tendrían como finalidad mejorar las condiciones materiales y psicológicas en que se produce ese aprendizaje. Gran parte de las estrategias incluidas dentro de esta categoría tiene que ver con la disposición afectiva y motivacional del sujeto hacia el aprendizaje.

La importancia de los componentes afectivo-motivacionales en la conducta estratégica es puesta de manifiesto por la mayor parte de los autores que trabajan en este campo. Todos coinciden en manifestar que los motivos, intenciones y metas de los estudiantes determinan en gran medida las estrategias específicas que utilizan en tareas de aprendizaje particulares. Por eso se entiende que la motivación es un componente necesario de la conducta estratégica y un requisito previo para utilizar estrategias.

Todo esto indica que los estudiantes suelen disponer de una serie de estrategias para mejorar el aprendizaje, aunque la puesta en marcha de las mismas depende, entre otros factores, de las metas que persigue el alumno, referidas tanto al tipo de metas académicas (por ejemplo, metas de aprendizaje - metas de rendimiento) como a los propósitos e intenciones que guían su conducta ante una tarea de aprendizaje en particular.

\section{Según Román y Gallegos (1994) ${ }^{9}$ los tipos de estrategias de aprendizaje son:}

1.Estrategias de adquisición de información, de acuerdo al modelo de Atkinson y Shiffrin, para adquirir información es necesario los procesos atencionales, que son los encargados de seleccionar, transformar y transportar la información desde el medio ambiente al Registro Sensorial. Luego se ponen en marcha los procesos de repetición, encargados de llevar la información (transformarla y transportarla), junto con los atencionales y en interacción con ellos, desde el Registro Sensorial a la Memoria a Corto Plazo (MCP). En el ámbito de la adquisición de la información se han constatado dos tipos 
de estrategia de procesamiento (véase cuadro 1):

1.1 Estrategias atencionales, los procesos de enseñanza - aprendizaje se dirigen a favorecer los procesos atencionales y, mediante ellos, el controlo dirección de todo el sistema cognitivo hacia la información relevante de cada contexto. Dentro de las atencionales, se distinguen las estrategias de Exploración, cuando los conocimientos previos sobre el material verbal que se haya de aprender sea grande, cuando las «metas u objetivos» del aprendizaje no sean claros, y cuando el material verbal disponible para el estudio no esté «bien organizado». Y de Fragmentación cuando los «conocimientos previos» acerca del tema objeto de aprendizaje sea pobre, cuando las «metas u objetivos» estén claros, y cuando el material de trabajo esté bien organizado.

1.2 Estrategias de repetición, la repetición tiene la función de hacer durar y/o hacer posible y facilitar el paso de la información a la Memoria a Largo Plazo (MLP). Se emplean tales estrategias para repasar una y otra vez estrategias para repasar una y otra vez el material verbal a aprender, de las diversas formas que es posible hacerla, y utilizando, simultáneamente, los receptores más variados: vista (lectura), oído (audición si se ha grabado anteriormente), cinestesia-motriz (escribiendo), boca (diciendo en alta voz) y/o mente (pensando en ello, «diciéndolo» mentalmente). La escala identifica operativamente tres tácticas de repetición: repaso en voz alta, repaso mental y repaso reiterado.

2. Estrategias de codificación de información, Se refiere al proceso cognitivo encargado de la elaboración y organización de la información, conectándola con conocimientos previos e integrándola en estructuras de significado más amplias que forman la base del conocimiento, es decir el paso de la memoria de corto plazo (MCP) a la memoria de largo plazo (MLP).

2.1 Estrategias nemotécnicas, utilizar nemotécnicas para un aprendizaje supone una codificación superficial o elemental, sin demasiada dedicación de tiempo y esfuerzo al procesamiento. La información puede ser reducida a una palabra-clave (Raugh y Atkinson las popularizaron en al aprendizaje de vocabulario de una lengua extranjera), o pueden organizarse los elementos a aprender en forma de siglas, rimas, frases, etc., es decir, utilizando medios nemotécnicos.

2.2 Estrategias de elaboración, Weinstein y Mayer (1986)2 distinguen dos niveles de elaboración: el simple, basado en la asociación intra material a aprender, y el complejo que lleva a cabo la integración de la información en los conocimientos previos del individuo. El almacenamiento duradero parece depender más de la elaboración y/u organización de la información que de las nemotécnicas.

2.3 Estrategias de organización, son un tipo especial de elaboración o una fase superior de la misma. Hacen que la información sea todavía más significativa (relacionada con lo que el sujeto sabe e integrada en su estructura cognitiva) y más manejable (reducida de tamaño) para el estudiante.

3.Estrategias de Recuperación de información, Se refiere a las estrategias que favorecen la búsqueda de información en la memoria y la generación de respuesta e incluyen:

3.1 Estrategias de búsqueda, se hallan básicamente condicionadas por la organización de los conocimientos en la memoria, resultado a su vez de las estrategias de codificación. La calidad de los «esquemas» (estructuras abstractas de conocimientos) elaborados constituyen, pues, el campo de búsqueda que tienen lugar en un individuo y guardan correspondencia con los utilizados por el mismo para la codificación. Las estrategias de búsqueda sirven para facilitar el control o la dirección de la búsqueda de palabras, significados y representaciones conceptuales o icónicas en la MLP. Fundamentalmente, en este campo se han venido constatando dos estrategias: 
3.2 Estrategias de generación de respuesta, La generación de una respuesta debidamente realizada puede garantizar la adaptación positiva que se deriva de una conducta adecuada a la situación. Las tácticas para ello pueden adoptar una disposición secuencial, como pueden ser la búsqueda de codificaciones, la búsqueda de indicios, la planificación de respuesta y la respuesta escrita.

4.Escala de Estrategias de Apoyo al procesamiento, Durante el tiempo que dura el procesamiento de información, otros procesos de naturaleza metacognitiva y no-cognitiva, los de apoyo, optimizan, son neutrales o entorpecen el funcionamiento de las estrategias cognitivas de aprendizaje. .por eso los alumnos también necesitan estrategias y tácticas que les ayuden a «manejar» sus procesos de apoyo. Las estrategias de apoyo, ayudan y potencian el rendimiento de las estrategias de adquisición (escala I), de las de codificación (escala II) y de las de recuperación (escala III), incrementando la motivación, la autoestima, la atención. Garantizan el clima adecuado para un buen funcionamiento de todo el sistema cognitivo. De ahí que para llevar a cabo el procesamiento y recuperación de información sea imprescindible su identificación y correcto manejo.

4.1 Estrategias metacognitivas, las estrategias metacognitivas suponen y apoyan, por una parte, el conocimiento que una persona tiene de los propios procesos, en general, y de estrategias cognitivas de aprendizaje, en particular y, por otra, la capacidad de manejo de las mismas.

4.2 Estrategias socioafectivas, Es indudable que los factores sociales están presentes en el nivel de aspiración, autoconcepto, expectativas de autoeficacia, motivación, etc., incluso en el grado de ansiedad/relajación con que el alumno se dispone a trabajar. Ha sido la dificultad para separar todos estos campos, y no la decisión de ignorarlos, lo que ha determinado la etiqueta. Un análisis sobre la naturaleza de todas ellas puede sugerir la afirmación de que, de una u otra forma, se dirigen a controlar, canal izar o reducir la ansiedad, los sentimientos de incompetencia, las expectativas de fracaso, la autoeficacia, el locus de control, la autoestima académica, etc., que suelen aparecer cuando los estudiantes se enfrentan a u una tarea compleja, larga y difícil de aprendizaje. Para Biggs (1993)10 el estudio de las estrategias de aprendizaje se sustenta en dos afrontes teóricos:

a. El modelo de procesamiento de la información que intenta establecer el significado que tienen las estrategias de aprendizaje para el procesamiento de la información.

b. El modelo orientado al contexto, y que se sustenta en el estudio de la fenomenología de la conducta real de estudio y aprendizaje en las instituciones (Yussen, 1985)11.

La reflexión, según Boud, Keogh \& Walker $(1988)^{12}$, es un activo y cuidadoso proceso de pensamiento, y constituye una actividad en la cual las personas recuerdan y evalúan sus experiencias

Para De La Harpe \& Radloff (1998)13, la reflexión implica una forma de aprendizaje a través del pensamiento activo que se sustenta sobre los aprendizajes pasados, de manera que permiten anticipar el futuro rendimiento y constituye un importante aspecto del aprendizaje autorregulado, de modo que los estudiantes que utilizan la reflexión en sus aprendizajes, presentan una mejor comprensión de los procesos que los regulan, por lo cual pueden ser capaces de ejercer un mejor control sobre su propio aprendizaje.

Por otro lado para Mathison (1994)14, la reflexión se sustenta en las nociones de pensamiento reflexivo de Dewey y la teoría Piagetana del desarrollo cognitivo, por lo que reflexión incluye las capacidades para auto-observarse, analizar las experiencias pasadas, evaluar su significado y desarrollar un plan de acción basado en el análisis y la reflexión.

La reflexión para Biggs \& Moore (1993)15, está estrechamente unida a la metacognición (pues ésta, incluye aprendizajes centrados en los procesos cognitivos, así como el uso de procedimientos que controlan y estimulan dichos procesos. La 
metacognición implica la atención en el conocimiento de la cognición que involucra variables relacionadas a las personas y las estrategias de trabajo y el control y regulación de la cognición, haciendo uso de la planificación, monitoreo, evaluación y el pensamiento adaptativo.

Angelo $(1991)^{16}$ indica que los estudiantes para lograr alcanzar aprendizajes independientes necesitan aprender a ser autoreflexivos para comprender la forma como piensan y se desarrollan sus creencias. Además indica que la reflexión es esencial para aprender y es el mecanismo central que facilita el desarrollo y el cambio cognitivo.

Para Ewell (1997)17, la reflexión es un proceso mental, el cual al ser aplicado al proceso de aprendizaje, puede motivar a los alumnos a usar el pensamiento crítico para examinar la información presentada, cuestionar su validez y sacar conclusiones basadas en sus ideas resultantes. Estos procesos permiten que los alumnos limiten las posibles soluciones y formulen eventualmente una conclusión. El resultado de éste proceso es una mejor comprensión del concepto.

Las situaciones de aprendizaje efectivo requieren de un determinado tiempo para pensar. Los alumnos también pueden reflexionar acerca de lo que ellos utilizan cuando efectúan sus procesos de pensamiento, de manera que pueden determinar que estrategias funcionan mejor, de manera que pueden aplicar dicha información para realizar aprendizajes futuros.

La reflexión según Ewell (1997)17, puede formar parte del aprendizaje autorregulado, el cual ocurre cuando el alumno ejecuta y mantiene conductas sistemáticamente orientadas hacia el cumplimiento de objetivos; en este caso, la adquisición de conocimientos implica actividades dirigidas por metas, que los estudiantes son capaces de poner en marcha, mantener y modificar. Para Schunk, (1990) ${ }^{16}$, una meta es lo que un individuo está intentando conseguir de forma consciente, y formular una meta implica establecerla, y modificarla si se considera necesario, la meta sería una representación cognitiva de lo que queremos que suceda (o de lo que deseamos evitar) en el futuro.

En el ámbito escolar, las metas pueden tener distintos orígenes: el alumno puede crear sus propias metas, adopta las metas propuestas por otros, desarrolla metas con otros, o recibe metas impuestas externamente tal como lo indican Karniol y Ross (1996) ${ }^{19}$. El alumno no sólo debe asumir las distintas metas que se le plantean y seleccionarlas, sino que puede decidir qué es lo importante para él y formular sus propios objetivos de actuación. Para esto se requiere que el sujeto se conozca a sí mismo y que tenga unas expectativas realistas sobre lo que es capaz de llevar a cabo; también debe ser consciente de sus preferencias, intereses y valores, para lo que se necesita un cierto nivel de autoconciencia (Patalano y Seifert, 1997) ${ }^{20}$. A partir de este autoconocimiento, el sujeto dispone de instrumentos para seleccionar o plantear sus propias metas personales y significativas; asimismo, puede evaluar sus posibilidades de éxito o fracaso, generar expectativas de resultados, y comprometerse a alcanzar sus metas. En esta fase, además del autoconocimiento, es fundamental la capacidad para reflexionar y evaluar la importancia y la relevancia de los objetivos elegidos, primer paso esencial para generar compromisos de actuación duraderos y sentimientos positivos hacia la consecución de las metas.

Como indican Vermunt (1995) ${ }^{21}$ y Justicia (1996) ${ }^{22}$, el aprendizaje autoregulado en los estudiantes en general, parece estar constituido por tres aspectos básicos:

a. Las estrategias metacognitivas de autoregulación, planificacion, autocontrol y consciencia del propio estudio y sus efectos.

b. Las estrategias cognitivas, que constituyen procedimientos intencionales que permiten al estudiante tomar decisiones respecto a su estudio y rendimiento.

c. Las estrategias de apoyo, que incluyen a la motivación afecto y actitudes, y que están relacionadas al control personal del esfuerzo, de la persistencia y a promover las 
condiciones que faciliten el estudio.

El estudio de la reflexión activa en la actualidad ha llevado al desarrollo de diferentes campos de investigación, como es el caso del estudio de la metacognición del aprendizaje, especialmente en lo que se refiere al rol que desempeña la percepción de la autoeficacia en la regulación de las actividades metacognitivas, donde Zimmerman Bandura y Martínez Pons $(1992)^{23}$ indican que la eficacia en la autoregulación del aprendizaje afecta las metas que se pueden establecer los estudiantes por sí mismos. También se ha estudiado el rol que desempeña la reflexión en la transformación de las experiencias de los sujetos en aprendizajes, como es el caso de Boud Keogh \& Walker (1988) ${ }^{12}$ y el estudio de las características de las estrategias de procesamiento del aprendizaje, las cuales según Richardson $(1995)^{24}$, incluye:

a. El enfoque profundo del aprendizaje, que tiende a producir elementos que representan un entendimiento más sustancial de las tareas de aprendizaje, en razón del desarrollo personal y la búsqueda del significado y la integración de un nuevo conocimiento en uno ya adquirido, notándose que para Gibbs $(1992)^{25}$, la reflexión es una estrategia que favorece el enfoque profundo del aprendizaje, resaltando como sus principales componentes la conciencia de lo que se aprende y la conciencia de cómo se aprende.

b. El enfoque superficial del aprendizaje, que tiende a producir elementos que representan un bajo entendimiento y es de carácter reproductivo, debido a la memorización desorganizada de hechos y de conceptos.

Existe una gran cantidad de trabajos empíricos sobre el aprendizaje autoregulado que se han realizado con escolares, notándose que la instrucción en la escuela tradicional se caracteriza por la existencia de un fuerte control externo en el cual el profesor asume la responsabilidad de regular el aprendizaje de sus alumnos (Vermunt, 1995) ${ }^{21}$

Como antecedente encontramos que Sobral (1998)26 construyó la escala de Reflexión en el Aprendizaje para evaluar la capacidad de aprendizaje autodirigido de los estudiantes universitarios de primeros ciclos, alcanzando resultados significativos. En nuestro contexto no existen estudios similares y este concepto ha sido evaluado de forma diferente bajo' el concepto de estilos de aprendizaje de Kolb, habiéndose desarrollado los estudios de Eisler $(1989)^{27}$ y Escurra $(1991)^{28}$.

La reflexión en el campo de la Psicología Educativa actual, constituye un aspecto muy importante en el análisis de los procesos de aprendizajes cognitivos, pues como mencionan Hammond y Collins $(1991)^{29}$, la reflexión es una estrategia básica para desarrollar el aprendizaje autodirigido, motivo por el cual puede influir en el rendimiento escolar.

Respecto a los estudios sobre el rendimiento escolar, tal como indica Grouws (1992)30 hasta la década de los 80 la mayoría de investigaciones relacionadas con el rendimiento académico se hacia con estudios correlacionales, calculándose ecuaciones predictoras de regresión y analizando la influencia de variables sueltas en el rendimiento en general y en el rendimiento en matemáticas. La revisión bibliográfica de estudios recientes reportados por Page $(1990)^{31}$, Cuevas Renaud y Aguilar Villalobos (1999) ${ }^{32}$ y Arrieta $(2000)^{33}$ reportan la existencia de modelos teóricos concretos y el uso del método de ecuaciones estructurales para avanzar en la explicación del rendimiento académico de los alumnos.

Respecto a los estudios relacionados con el rendimiento efectuados en nuestro medio, la bibliografía reporta que, Aliaga, Ponce, Gutiérrez, Díaz, Reyes y Pinto $(2001)^{34}$ analizaron en estudiantes de psicología de la UNMSM, las relaciones entre el autoconcepto académico, los rasgos, el respeto por las normas y sensibilidad, la actitud hacia las matemáticas y la estadística con el rendimiento en los cursos de matemática y estadística. Los resultados alcanzados indicaron que el valor de la tarea, las estrategias de aprendizaje 
de metacognición y autointerrogatorio y la búsqueda de ayuda influyeron en el rendimiento en los cursos de matemáticas y estadísticas.

Por otro lado García, Ore llana y Canales (2002)35 evaluaron los factores que estaban asociados al rendimiento académico en los estudiantes de psicología de la UNMSM, encontrando que existieron correlaciones negativas entre la orientación a metas intrínsecas y la ansiedad con -el rendimiento académico y una asociación positiva entre la estrategia, la constancia y el rendimiento.

Finalmente Aliaga y Giove (2003)36 analizaron las diferencias en el MSLQ y el LASSI entre los alumnos del quinto año de secundaria con autoevaluación alta y baja del rendimiento académico en matemática, observando que 8 de las 10 escalas del LASSI, 7 de las 12 escalas del MSLQ, la autoeficacia para el aprendizaje y la ansiedad ante los exámenes influyen en el rendimiento.

Es por lo anteriormente expuesto que la finalidad de la presente investigación fue estudiar la influencia de las estrategias de aprendizaje y la reflexión activa sobre el rendimiento escolar de los alumnos de quinto año de secundaria de la ciudad de Lima Metropolitana. 


\section{MATERIALES Y MÉTODOS}

La presente investigación, corresponde en un primer momento a un estudio correlacional pues se trató de estudiar el efecto de las estrategias de aprendizaje y la reflexión activa sobre el rendimiento escolar de los alumnos, en un segundo momento se trabajó con un diseño descriptivo comparativo pues se trató de estudiar la existencia de diferencias en las estrategias de aprendizaje, la reflexión activa y el rendimiento escolar según el tipo de colegio al que pertenecen los alumnos y el sexo (Sánchez y Reyes, 2002).

El universo de investigación estuvo conformado por los alumnos de quinto año de secundaria matriculados en el año 2003 en los colegios estatales y particulares de Lima .Metropolitana. Para la obtención de la muestra se aplicó un procedimiento de muestreo probabilístico por conglomerdos Bi-etápico, en la primera etapa en cada una de las UGEL, se eligieron de forma aleatoria los colegios a ser incluidos en el estudio y posteriormente en cada colegio se eligió de manera aleatoria el aula de quinto año de secundaria que fue evaluada.

La muestra estuvo constituida por 1200 alumnos con edades que fluctuaban entre los 15 y los 18 años, El promedio de Edad fue de 16.1 y se obtuvo una desviación estándar de 0.86. La mayor cantidad correspondió a los que tuvieron 16 años (48.6 \%). El $52.0 \%$ fueron mujeres ye148.0 \% fueron varones. Respecto a la gestión del colegio existieron más alumnos pertenecientes a colegios estatales $(53.8 \%)$ que a colegios particulares $(46.21 \%)$. La mayoría de alumnos eran de Lima (61.1 $0 \%)$.

Los instrumentos utilizados fueron:

Escala de Reflexión Activa, fue desarrollada a partir del Inventario de Estilos de Aprendizaje de Kolb, adaptada por Escurra (1992) ${ }^{38}$ y la Escala de Reflexión sobre el Aprendizaje (Escurra, 2002) ${ }^{39}$. La escala estuvo constituida por 6 afirmaciones acerca de diferentes aspectos asociados a la aplicación de la reflexión de forma activa en el aprendizaje. En la escala, todos los enunciados se califican sobre la base de un sistema tipo likert con 5 puntos de calificación, los cuales incluyen las siguientes opciones; No es característico (1), Poco característico (2), Algo característico (3) Característico (4) y Muy Característico (5).

El instrumento puede ser aplicado tanto de forma individual como colectiva y está diseñado para ser administrado a grupos de adolescentes por el tipo de afirmaciones que contiene. El tiempo de duración de la aplicación del instrumento es de aproximadamente entre 5 y 10 minutos. Para la construcción del instrumento se estudio la validez de constructo y la confiabilidad por consistencia interna a través del coeficiente Alfa de Cronbach.

Escalas de Estrategias de Aprendizaje (ACRA), desarrollado originalmente por Román Sánchez y Gallego (1984) ${ }^{9}$. Está integrado por varios ítemes para cada estrategia que presentan una serie de afirmaciones, en torno al uso de la misma, con respecto a las cuales los alumnos se han de pronunciar con cuatro posibles respuestas: nunca o casi nunca, algunas veces, muchas veces y siempre o casi siempre. El instrumento está constituido por cuatro escalas independientes que evalúan el uso que habitualmente hacen los estudiantes de ellas, las cuales son:

Escala I, mide las estrategias de adquisición de información (integra estrategias atencionales: exploración, subrayado lineal, subrayado idiosincrásico, epigrafiado, y estrategias de repetición: repaso en voz alta, repaso mental y repaso reiterado).

Escala II, mide las estrategias de codificación o almacenamiento de información, que incluye las estrategias de mnemotecnias, las estrategias de elaboración (relaciones 
intracontenido, relaciones compartidas, imágenes, metáforas, aplicaciones, autopreguntas, paráfrasis) y las estrategias de organización (agrupamientos, secuencias, mapas conceptuales y diagramas).

Escala III, mide las estrategias de recuperación de información como son las estrategias de búsqueda (búsqueda de codificaciones, búsqueda de indicios) y las estrategias de generación de respuesta (planificación de respuesta y respuesta escrita).

Escala IV, mide las estrategias de apoyo al procesamiento, que incluye a las estrategias metacognitivas(autoconocimiento, automanejo/ planificación automanejo /regulación y evaluación) y las estrategias socioafectivas (autoinstrucciones, autocontrol, contradistractoras, interacciones sociales, motivación intrínseca y extrínseca y motivación de escape).

Las escalas ACRA pueden ser aplicadas en distintas fases (evaluación inicial, final o de seguimiento) y tipos de intervención psicoeducativa: (a) preventiva (entrenar en determinada estrategia general tras constatar que su carencia o su incorrecta utilización afecta negativamente al rendimiento de los estudiantes) o (b) optimizándola (entrenar en determinada estrategia a un alumno que aunque ya la utiliza, se busca que la pueda automatizar).

La prueba puede ser utilizada para evaluar a los alumnos que estudian secundaria y también puede ser ampliado a estudios superiores y puede ser aplicada tanto de forma individual como colectiva. Los autores reportan que la aplicación completa puede durar 50 minutos. Siendo el tiempo estimado para la aplicación de cada escala el siguiente: Escala I (10 minutos), Escala II (15 minutos), Escala III (8 minutos) y Escala IV (12 minutos).

En el Perú la escala ACRA fue adaptada por Cano (1996)40 en el desarrollo de su tesis sobre las Estrategias metacognitivas y cognitivas en el aprendizaje en alumnos del quinto de secundaria de NSE alto y medio alto en Lima Metropolitana, obteniendo resultados psicométricos adecuados, pero a pesar de ello y siguiendo la propuesta de Marín (1986)41 debido a la antigüedad del estudio se optó por realizar los análisis psicométricos respectivos de la validez y confiabilidad.

Previa elaboración de los manuales de codificación y calificación, la información fue procesada utilizando el paquete estadístico Spss FWv. 12. El análisis cuantitativo de los datos fue procesado en tres etapas utilizándose una combinación de estadística descriptiva y procedimientos multivariados. En la Etapa I se realizó el análisis Psicométrico de los instrumentos del estudio calculándose las correlaciones ítem test corregidas y los coeficientes Alfa de Cronbach, así como la validez de constructo a través del análisis factorial confirmatorio. En la Etapa II se realizó un análisis descriptivo de las variables seleccionadas para el estudio, efectuándose el análisis de la normalidad de los puntajes a través de la prueba de Kolmogorov-Smirnov. En la Etapa 111 se realizó el análisis inferencial del contraste de hipótesis, para ello se utilizó un modelo de ecuaciones estructurales para construir un análisis de caminos (path analysis) del efecto de las Estrategias de Aprendizaje y la Reflexión Activa sobre el rendimiento escolar, que constituye una variable latente a partir de las notas de matemáticas y comunicación. También se efectuaron las comparaciones de medias de las variables estudiadas según sexo y gestión del colegio, adicional mente se analizó el tamaño del efecto y la potencia estadística de las pruebas aplicadas.

El análisis de la información se realizó con los paquetes Spss FW v.12, para los análisis psicométricos de confiabilidad, las estadísticas descriptivas, la prueba de bondad de ajuste a la curva normal de Kolmogorov-Smirnov y las pruebas z para grupos independientes. Con los programas AMOS 5.0 (Arbuckle, 2000; 2004) ${ }^{42.43}$ Y LISREL 8.54 Obreskog \& Sbrbom, 2004) ${ }^{44}$ se efectuaron los análisis factoriales confirmatorios y el análisis de ecuaciones estructurales. Para el estudio del tamaño del efecto y la potencia de 
las pruebas estadísticas se utilizó el programa Power and Precisión 2.0 (Borenstein, Rothstein, \& Cohen, 2004) ${ }^{45}$

Los instrumentos se aplicaron de manera que se pudo identificar a los participantes a fin de obtener la información respectiva del rendimiento escolar, pero se les aseguro la confidencialidad de los resultados del estudio. Antes de la evaluación se realizó una breve explicación de la finalidad del estudio a los alumnos a fin de motivar su participación, dejando clara la completa libertad de participar o no en el estudio. 


\section{RESULTADOS}

\section{Etapa I. Análisis psicométrico Escala de Reflexión Activa}

Los resultados del desarrollo de la construcción de la Escala de Reflexión Activa en lo que concierne al análisis psicométrico de los ítemes del instrumento, efectuados a través del coeficiente de correlación ítem-test corregido, presentados en la Tabla 1 permiten observar que se obtienen correlaciones que fluctúan entre $\mathrm{r}=0.56$ (Item 1) $\mathrm{Y} \mathrm{r}=0.80$ (Item 4)/ los cuales son estadísticamente significativos $(\mathrm{p}<.05) \mathrm{Y}$ a la vez que superan el criterio de $r>$ 0.20 propuesto por Kline (1995)46, lo que permite que todos los ítems fueron relevantes para la conformación de la escala. El análisis de la confiabilidad por consistencia interna, realizado a través del coeficiente Alfa de Cronbach, alcanzó un valor de 0.89/ por lo que se puede concluir que la escala de Reflexión en el Aprendizaje permite obtener puntajes confiables.

\section{Tabla 1.- Análisis Psicométrico de la Escala de Reflexión Activa}

\begin{tabular}{|c|c|c|c|}
\hline Ítem & Media & D.E & ritc. \\
\hline 1 & 3.35 & 1.03 & $0.56^{*}$ \\
2 & 3.92 & 1.05 & $0.60^{*}$ \\
3 & 3.96 & 1.06 & $0.78^{*}$ \\
4 & 3.48 & 1.07 & $0.80^{*}$ \\
5 & 3.71 & 0.99 & $0.76^{*}$ \\
6 & 3.98 & 1.56 & $0.77^{*}$ \\
\hline \multicolumn{3}{|c}{ Coeficiente alfa de Cronbach $=0.89^{*}$} \\
\hline
\end{tabular}

El estudio de la Validez de Constructo efectuado a través del Análisis Factorial Confirmatorio, con base a la aplicación de los programas AMOS 5.0 (Arbuckle, 2000; $2004)^{42.43}$ y LISREL 8.54 (Joreskog \& Sorbom, 2004)44 tomó en cuenta las recomendaciones propuestas por Byrne $(1989,1998)^{47.48}$ quien propone la necesidad de evaluar el modelo teórico propuesto y un modelo alternativo diferenciado que sirva de criterio de contraste.

Los resultados observados en la tabla 2 , donde se incluye el modelo teórico propuesto que indica la existencia de una sola dimensión latente, permitió observar que el modelo propuesto alcanza en el test de Bondad de Ajuste Chi-Cuadrado Mínimo (Cmin) un valor de $1.15 /$ el cual con 1 grado de libertad alcanza una probabilidad de 0.241 , lo que nos indica que el modelo es adecuado y obtiene resultados superiores al modelo Independiente. Además la revisión de los hallazgos del análisis del índice residual de la raíz cuadrada media que evalúa la aproximación de la matriz de covarianzas teórica con la matriz observada, alcanzó un valor pequeño $(\mathrm{RMR}=0.05)$ y los análisis complementarios de la bondad de Ajuste a través del índice de Ajuste (GFI =0.97) y el índice de Ajuste Ponderado (AGFI $=0.96$ ) alcanzaron valores óptimos, estos hallazgos permitieron corroborar lo pertinente de aceptar el modelo de un factor. Por lo que se concluye que la escala de Reflexión Activa presenta validez de Constructo. 
Tabla 2.- Análisis de la Validez de Constructo de la Escala de Reflexión Activa a través del Análisis Factorial Confirmatorio

\begin{tabular}{|c|c|c|}
\hline Datos & Modelo de 1 factor & Modelo independiente \\
\hline Parámetros & 12 & 6 \\
Chi-cuadrado mínimo & 1.15 & 27.85 \\
G.L & 1 & 6 \\
P & 0.241 & 0.000 \\
Chi- & 1.15 & 4.65 \\
cuadrado/mínimo/G.L & 0.05 & 0.13 \\
RMR & 0.97 & 0.89 \\
GFI & 0.96 & 0.59 \\
AGFI & & \\
& & \\
\hline
\end{tabular}

$\mathrm{N}=1200$

\section{Escalas de estrategias de aprendizaje (ACRA)}

El análisis de ítemes efectuado por medio del coeficiente de correlación ítem-test corregido, presentado en la Tabla 3, permite apreciar que se obtuvieron correlaciones que son estadísticamente significativas $(p<0.05)$, las cuales superan el criterio de $r>$ 0.20 propuesto por Kline (1995)46, por lo cual se puede afirmar que todos los ítemes son relevantes para la escala. La revisión de la confiabilidad por consistencia interna, calculado a través del coeficiente Alfa de Cronbach, indica que se obtiene un valor de 0.77, por lo que se puede concluir que la escala de Adquisición de la Información permite obtener puntajes confiables.

Tabla 3.- Análisis Psicométrico de la Escala de Adquisición de la información del ACRA

\begin{tabular}{|c|c|c|c|}
\hline Item & Media & D.E: & ritc \\
\hline I & 2.50 & 1.03 & 0.30 \\
\hline 2 & 2.55 & 0.93 & 0.36 \\
\hline 3 & 2.40 & 0.92 & 0.29 \\
\hline 4 & 2.70 & 0.99 & 0.37 \\
\hline 5 & 2.32 & 1.06 & 0.28 \\
\hline 6 & 2.37 & 1.06 & 0.31 \\
\hline 7 & 2.42 & 0.97 & 0.33 \\
\hline 8 & 2.36 & 1.00 & 0.43 \\
\hline 9 & 2.32 & 0.95 & 0.38 \\
\hline 10 & 3.04 & 0.93 & 0.28 \\
\hline 11 & 3.26 & 0.88 & 0.35 \\
\hline 12 & 2.60 & 1.02 & 0.35 \\
\hline 13 & 0.68 & 1.02 & 0.46 \\
\hline 14 & 2.95 & 0.91 & 0.35 \\
\hline 15 & 2.97 & 0.93 & 0.42 \\
\hline 16 & 2.24 & 0.89 & 0.28 \\
\hline 17 & 2.04 & 0.90 & 0.26 \\
\hline 18 & 2.15 & 0.95 & 0.44 \\
\hline 19 & 2.80 & 0.96 & 0.33 \\
\hline \multicolumn{4}{|c|}{ Coeficiente Alfa de Cronbach $=0.77 *$} \\
\hline
\end{tabular}


El análisis de ítemes de la Escala de Codificación de la información (Ver Tabla 4), permite observar que se alcanzaron correlaciones que fluctúan entre $\mathrm{r}=0.30 \mathrm{y} \mathrm{r}=0.74$, siendo en todos los casos estadísticamente significativas a $\mathrm{p}<.05$, por lo cual se puede indicar que todos los ítemes son relevantes para la escala. El análisis de la confiabilidad por consistencia interna, calculado a través del coeficiente Alfa de Cronbach, asciende a 0.91, por lo que se puede concluir que la escala permite obtener puntajes confiables.

Tabla 4.- Análisis Psicométrico de la Escala de Codificación de la Información del ACRA

\begin{tabular}{|c|c|c|c|c|c|c|c|}
\hline Ítem & Media & O.E: & ritc & Ítem & Media & O.E: & ritc \\
\hline 1 & 2.11 & 0.93 & $0.40 *$ & 24 & 2.33 & 1.01 & $0.47 *$ \\
\hline 2 & 2.53 & 0.96 & $0.44 *$ & 25 & 2.90 & 0.98 & $0.34 *$ \\
\hline 3 & 2.60 & 0.87 & $0.35 *$ & 26 & 2.03 & 0.93 & $0.30 *$ \\
\hline 4 & 2.34 & 0.87 & $0.34 *$ & 27 & 2.45 & 0.83 & $0.46 *$ \\
\hline 5 & 2.59 & 0.88 & $0.40 *$ & 28 & 2.63 & 0.85 & $0.47 *$ \\
\hline 6 & 2.58 & 0.88 & $0.40 *$ & 29 & 2.56 & 0.94 & $0.46 *$ \\
\hline 7 & 2.67 & 0.90 & $0.43 *$ & 30 & 2.89 & 0.92 & $0.45 *$ \\
\hline 8 & 2.70 & 0.99 & $0.33 *$ & 31 & 2.56 & 0.97 & $0.38 *$ \\
\hline 9 & 2.83 & 1.02 & $0.31 *$ & 32 & 2.50 & 0.96 & $0.47 *$ \\
\hline 10 & 2.64 & 0.98 & $0.38 *$ & 33 & 2.12 & 0.94 & $0.36 *$ \\
\hline 11 & 2.56 & 0.93 & $0.36 *$ & 34 & 2.33 & 0.96 & $0.43 *$ \\
\hline 12 & 2.39 & 1.01 & $0.33 *$ & 35 & 2.35 & 0.88 & $0.44 *$ \\
\hline 13 & 2.74 & 1.02 & $0.35 *$ & 36 & 2.46 & 0.85 & $0.43 *$ \\
\hline 14 & 2.27 & 0.90 & $0.44 *$ & 37 & 2.02 & 0.90 & $0.49 *$ \\
\hline 15 & 2.39 & 0.92 & $0.42 *$ & 38 & 2.11 & 0.88 & $0.41 *$ \\
\hline 16 & 2.21 & 0.94 & $0.43 *$ & 39 & 2.66 & 0.98 & $0.42 *$ \\
\hline 17 & 2.76 & 0.90 & $0.40 *$ & 40 & 1.91 & 0.87 & $0.38 *$ \\
\hline 18 & 2.45 & 0.88 & $0.40 *$ & 41 & 1.97 & 0.85 & $0.42 *$ \\
\hline 19 & 2.48 & 0.91 & $0.46 *$ & 42 & 2.40 & 0.93 & $0.35 *$ \\
\hline 20 & 2.13 & 0.87 & $0.38 *$ & 43 & 2.18 & 0.97 & $0.35 *$ \\
\hline 21 & 2.44 & 0.89 & $0.36 *$ & 44 & 1.70 & 0.86 & $0.35 *$ \\
\hline 22 & 2.33 & 0.95 & $0.40 *$ & 45 & 2.40 & 0.94 & $0.37 *$ \\
\hline 23 & 2.70 & 0.90 & $0.43 *$ & 46 & 2.45 & 1.02 & $0.74 *$ \\
\hline
\end{tabular}

$* p<.05$

$N=1200$ 
Tabla 5.- Análisis Psicométrico de la Escala de Recuperación de la información del ACRA

\begin{tabular}{|l|l|l|l|}
\hline Item & Media & D.E: & ritc \\
\hline 1 & 2.71 & 0.91 & $0.47^{*}$ \\
2 & 2.31 & 0.94 & $0.40^{*}$ \\
3 & 2.70 & 0.92 & $0.47^{*}$ \\
4 & 2.92 & 0.88 & $0.47^{*}$ \\
5 & 2.82 & 0.89 & $0.49^{*}$ \\
6 & 2.70 & 0.92 & $0.48^{*}$ \\
7 & 2.59 & 0.90 & $0.45^{*}$ \\
8 & 2.57 & 0.94 & $0.45^{*}$ \\
9 & 2.79 & 0.95 & $0.47^{*}$ \\
10 & 2.78 & 0.90 & $0.52^{*}$ \\
11 & 3.03 & 0.87 & $0.47^{*}$ \\
12 & 2.95 & 0.93 & $0.49^{*}$ \\
13 & 2.94 & 0.94 & $0.48^{*}$ \\
14 & 2.66 & 0.96 & $0.48^{*}$ \\
15 & 2.88 & 0.95 & $0.38^{*}$ \\
16 & 2.46 & 0.94 & $0.36^{*}$ \\
17 & 2.64 & 0.88 & $0.39^{*}$ \\
18 & 2.85 & 0.90 & $0.44^{*}$ \\
\multicolumn{2}{l|}{} & & \\
\hline Coeficiente Alfa de Crombach=0.86* & \\
*p<.05 & & \\
$\mathrm{N}=120$ &
\end{tabular}


La revisión del análisis de la Escala de Apoyo al Procesamiento (Ver Tabla 6), permite apreciar que se alcanzan correlaciones estadísticamente significativas y mayores que $\mathrm{r}-0.35$, las cuales superan el criterio establecido, cual permite indicar que todos los ítemes son pertinentes para conformar la escala. El análisis de la confiabilidad por consistencia interna, calculado a través del coeficiente Alfa de Cronbach, asciende a 0.89, por lo que se puede concluir que la escala permite obtener puntajes confiables.

Tabla 6.- Análisis Psicométrico de la Escala de Apoyo al Procesamiento del ACRA

\begin{tabular}{|c|c|c|c|}
\hline Item & Media & D.E: & ritc \\
\hline 1 & 2.49 & 0.90 & $0.54^{*}$ \\
\hline 2 & 2.68 & 0.88 & $0.40^{*}$ \\
\hline 3 & 2.55 & 0.86 & $0.48^{*}$ \\
\hline 4 & 2.54 & 0.92 & $0.44 *$ \\
\hline 5 & 2.76 & 0.95 & $0.43 *$ \\
\hline 6 & 2.72 & 0.96 & $0.48^{*}$ \\
\hline 7 & 2.88 & 0.89 & $0.52 *$ \\
\hline 8 & 2.61 & 0.89 & $0.56^{*}$ \\
\hline 9 & 2.71 & 0.93 & $0.46^{*}$ \\
\hline 10 & 2.61 & 0.93 & $0.44^{*}$ \\
\hline 11 & 2.87 & 0.97 & $0.38 *$ \\
\hline 12 & 2.42 & 0.97 & $0.36^{*}$ \\
\hline 13 & 2.49 & 0.87 & $0.45^{*}$ \\
\hline 14 & 2.63 & 0.84 & $0.50 *$ \\
\hline 15 & 2.76 & 0.92 & $0.51^{*}$ \\
\hline 16 & 2.79 & 0.96 & $0.49 *$ \\
\hline 17 & 3.01 & 0.93 & $0.41 *$ \\
\hline 18 & 2.71 & 0.91 & $0.48^{*}$ \\
\hline 19 & 2.31 & 0.94 & $0.52 *$ \\
\hline 20 & 2.70 & 0.92 & $0.56^{*}$ \\
\hline 21 & 2.92 & 0.88 & $0.46^{*}$ \\
\hline 22 & 2.82 & 0.89 & $0.44^{*}$ \\
\hline 23 & 2.70 & 0.92 & $0.38 *$ \\
\hline 24 & 2.59 & 0.90 & $0.36^{*}$ \\
\hline 25 & 2.57 & 0.94 & $0.45^{*}$ \\
\hline 26 & 2.79 & 0.95 & $0.50 *$ \\
\hline 27 & 2.78 & 0.90 & $0.52 *$ \\
\hline 28 & 3.03 & 0.87 & $0.56^{*}$ \\
\hline 29 & 2.95 & 0.93 & $0.46^{*}$ \\
\hline 30 & 2.94 & 0.94 & $0.44 *$ \\
\hline \multicolumn{4}{|c|}{ Coeficiente Alfa de Cronbach $=0.86^{*}$} \\
\hline
\end{tabular}


El análisis de la confiabilidad generalizada, tomando las escalas como ítemes, indica que se obtienen correlaciones ítem-test corregidas que son estadísticamente significativas y superiores a $\mathrm{r}=0.70$, lo cual indica que las escalas permiten obtener un valor total. Además se encuentra que la confiabilidad por consistencia interna obtenida con base al coeficiente alfa de Cronbach asciende a 0.89, lo cual indica que el ACRA permite obtener puntajes confiables

\section{Tabla 7.- Análisis Psicométrico de la Confiabilidad Generalizada del ACRA}

\begin{tabular}{|c|c|c|c|}
\hline Item & Media & D.E & ritc. \\
\hline Adquisición de la información & 2.41 & 10.53 & $0.74^{*}$ \\
Codificación de la información & 2.26 & 0.49 & $0.73^{*}$ \\
Recuperación de la información & 2.77 & 0.54 & $0.71^{*}$ \\
Apoyo al procesamiento & 2.69 & 0.57 & $0.72^{*}$ \\
\hline & & \\
\hline
\end{tabular}

El estudio de la Validez de Constructo también fue realizado a través del Análisis Factorial Confirmatorio, aplicándose los programas Lisrel 8.54 y Amos 5.0. Los resultados alcanzados en el modelo teórico propuesto de un factor, de acuerdo a las recomendaciones propuestas por Byrne $(1989,1998)^{47,48}$ se contrastó con un modelo un modelo alternativo

Los hallazgos del análisis de las escalas del ACRA (ver Tabla No. 8), permiten apreciar que el modelo de 1 factor presenta en el test de Bondad de Ajuste Chi-Cuadrado Mínimo (Cmin) un valor de 1.41, el cual con 1 grado de libertad alcanza una probabilidad de 0.234 , lo que nos indica que el modelo es adecuado. Además la revisión de los hallazgos del análisis del índice residual de la raíz cuadrada media que evalúa la aproximación de la matriz de covarianzas teórica con la matriz observada, presenta un valor pequeño (RMR $=$ 0.05) Y los análisis complementarios de la bondad de Ajuste a través del índice de Ajuste $(\mathrm{GFI}=0.99)$ Y el índice de Ajuste Ponderado (AGFI $=0.92)$ alcanzaron valores óptimos, estos hallazgos permitieron corroborar lo pertinente de aceptar el modelo de un factor. Por lo que se concluye que la escala ACRA presenta validez de Constructo.

Tabla 8.- Análisis de la Validez de Constructo de la Escala ACRA a través del Análisis Factorial Confirmatorio

\begin{tabular}{|c|c|c|}
\hline Datos & Modelo de 1 factor & Modelo independiente \\
\hline Parámetros & 9 & 4 \\
Chi-cuadrado mínimo & 1.41 & 36.62 \\
G.L & 1 & 6 \\
P & 0.234 & 0.000 \\
Chi- & 1.41 & 6.10 \\
cuadrado/mínimo/G.L & 0.05 & 0.26 \\
RMR & 0.99 & 0.72 \\
GFI & 0.92 & 0.54 \\
AGFI & & \\
\hline
\end{tabular}




\section{Etapa II. Análisis descriptivos}

Los resultados del análisis de la bondad de ajuste a la curva normal, fueron realizados a través de la prueba de Kolmogorov-Smirnov (ver Tabla 9), e indicaron que en todos los valores se obtuvieron estadísticos K-S Z que no son significativos, por lo que se pudo concluir que las distribuciones de los valores analizados se aproximaron adecuadamente a la distribución normal. Es por ello que se utilizaron contrastes estadísticos paramétricos en el análisis de los datos de la investigación (Siegel y Castellan, 1995) ${ }^{49}$.

Tabla 9.- Análisis de la Bondad de Ajuste a la Curva Normal de KolmogorovSmirnov de los valores estudiados

\begin{tabular}{|l|c|c|c|}
\hline Variables & Media & DS & Z \\
\hline ACRA & & & \\
Adquisición de la información & 2.461 & 0.43 & 1.08 \\
Codificación de la & 2.32 & 0.40 & 1.28 \\
Recuperación de la & 2.72 & 0.48 & 1.19 \\
Apoyo al procesamiento & 2.68 & 0.47 & 1.18 \\
Reflexión Activa & 24.29 & 3.96 & \\
Rendimiento Escolar & 12.56 & 1.59 & 1.17 \\
\hline
\end{tabular}

$N=1200$

\section{Etapa III. Análisis Inferencial del Contraste de Hipótesis}

Inicialmente se planteo la primera hipótesis teórica, la cual propuso que las estrategias de aprendizaje y la reflexión activa influyen positivamente sobre el rendimiento escolar en los alumnos del quinto año de secundaria. Para contrastarla se efectuó el análisis de Senderos (Path Analysis), asumiéndose como variables independientes a la reflexión activa y las estrategias de aprendizaje y como variable dependiente el Rendimiento Escolar, conformado por una variable latente obtenida a partir de las notas de matemáticas y las notas de comunicación.

El análisis de la validez del modelo se realizo a través del Modelo de Ecuaciones Estructurales y tomo en cuenta las recomendaciones de Byrne $(1989,1998)^{47.48}$ quien estableció para estos casos la necesidad de comparar el modelo teórico propuesto con dos modelos alternativos diferenciados, uno constituido por la intercorrelación entre todas las variables estudiadas y que constituye el Modelo Saturado y otro donde se incluye la menor cantidad posible de relaciones entre variables y que conforma el Modelo Independiente

El modelo es presentado en el gráfico 1 y los resultados se incluyen en la Tabla 10, donde se puede apreciar que el modelo Teórico propuesto un chi-cuadrado de 2.88 , que con 8 grados de libertad obtiene una probabilidad de 0.942 que no es estadísticamente significativa, lo cual indica la adecuación del modelo teórico respecto a los resultados obtenidos por el Modelo Saturado y el Modelo Independiente.

La revisión de los demás indicadores del modelo como es la relación entre el chicuadrado y los grados de libertad es menor a $3\left(\mathrm{X}^{2} / \mathrm{G} . \mathrm{L} .=0.36\right)$ la revisión de los hallazgos del análisis del índice residual de la raíz cuadrada media, que evalúa la aproximación de la matriz de covarianzas teórica con la matriz observada, alcanzó un valor pequeño $(\mathrm{RMR}=0.04) \mathrm{Y}$ el índice de la raíz cuadrada media del error de la aproximación, también alcanza un valor pequeño $(\mathrm{RMS} \& \backslash=0.01)$ alcanzaron valores pequeños. Los análisis complementarios del índice de ajuste $(\mathrm{GFI}=0.99) \mathrm{Y}$ el índice de ajuste ponderado $(\mathrm{AGFI}=0.99)$ son cercanos a 1 . En conclusión los resultados alcanzados permiten aceptar el modelo teórico propuesto. 
La revisión del modelo teórico propuesto permite observar que lo referido al modelo métrico, las correlaciones de las variables observadas con la variable latente son elevadas, pues las notas de matemáticas alcanzan un lambda $=0.80 \mathrm{Y}$ las notas de comunicación obtuvieron un Lambda $=0.79$.

El análisis de los efectos de las variables independientes permite notar que las estrategias y la reflexión activa ejercen un efecto positivo, es decir que los niveles elevados en dichas variables se corresponden con un rendimiento escolar más positivo. También se encuentra que el mayor efecto es producido por la estrategia de recuperación de la información y por la estrategia de apoyo al procesamiento es decir los aspectos más metacognitivos.

Tabla 10. Análisis del modelo de Senderos de toda la muestra

\begin{tabular}{|c|c|c|c|}
\hline Datos & $\begin{array}{c}\text { Modelo de } \\
\text { propuesto }\end{array}$ & $\begin{array}{c}\text { Modelo } \\
\text { independiente }\end{array}$ & $\begin{array}{c}\text { Modelo } \\
\text { independiente }\end{array}$ \\
\hline Chi-cuadrado & 2.88 & 0.000 & 752.97 \\
mínimo & 8 & 0 & 21 \\
G.L. & .942 & 28 & .000 \\
P & 20 & & 7 \\
Parámetros & 0.36 & 0.000 & 35.76 \\
X /G.L. & 0.04 & & 39.24 \\
RMR & 0.01 & & 359.00 \\
RMSEA & 0.99 & & 0.51 \\
GFI & 0.99 & & \\
AGFI & & & \\
\hline
\end{tabular}

Con relación a la segunda hipótesis teórica propuesta, en la cual se indica que existen diferencias en las estrategias cognitivas, la reflexión activa y el rendimiento escolar de los alumnos de quinto año de secundaria de acuerdo a la gestión del colegio de procedencia, fue real izado a través de la prueba $\mathrm{Z}$ de diferencia de medias, el cual es presentado en la Tabla 10, permite observar que existen diferencias estadísticas significativas a favor de los colegios particulares en; adquisición de la información $(Z=6.78 \mathrm{P}<.001)$, codificación de la información $(\mathrm{Z}=4.69 \mathrm{P}<.001)$, recuperación de la información $(\mathrm{Z}=2.23 \mathrm{P}<.05)$, apoyo al procesamiento $(\mathrm{Z}=3.44 \mathrm{P}<.01) \mathrm{Y}$ la reflexión activa $(\mathrm{Z}=7.51 \mathrm{P}<, 001)$.

Con la finalidad de obtener una evaluación más completa de los análisis estadísticos realizados, se calculó el tamaño del efecto, es decir cuan grandes son las diferencias que existen entre los grupos comparados y el poder estadístico de las pruebas utilizadas, es decir la probabilidad de evitar un error tipo II, que corresponde a la probabilidad de aceptar una hipótesis nula cuando realmente es cierta (Cohen, 1988, 1992; Aron y Aron 2001 y Clark, $2002)^{50,51,52,53}$

Los hallazgos presentados en la tabla 10, permiten apreciar que el tamaño del efecto, de las diferencias en los tipos de gestión de los colegio alcanzaron valores medios en la reflexión activa $(\mathrm{d}=0.44)$, Y en la adquisición de la información $(\mathrm{d}=0.40)$, mientras que presentaron valores pequeños en la codificación de la información $(\mathrm{d}=0.28)$, el apoyo al procesamiento de la información $(\mathrm{d}=0.19)$ Y la recuperación de la información $(\mathrm{d}=0.13)$, lo cual implica que las diferencias son significativas y adecuados en el estudio. En tanto que en el análisis del poder estadístico se aprecia que la reflexión activa y la adquisición de la información alcanzan niveles de 0.99 , en tanto que en la codificación de la información asciende a 0.94 , el apoyo al procesamiento es de 0.76 y la recuperación de la información a 0.61. De la revisión de los hallazgos encontrados se puede concluir que la segunda hipótesis es váli 
Tabla 10.- Prueba $Z$ de diferencia de medias de las Escalas de1 ACRA según Gestión del Colegio de Procedencia

\begin{tabular}{|c|c|c|c|c|c|c|c|}
\hline \multirow[b]{3}{*}{$\begin{array}{l}\text { Adquisición de la } \\
\text { información }\end{array}$} & \multicolumn{2}{|c|}{$\begin{array}{r}\text { No estatal } \\
\mathrm{n}=555\end{array}$} & \multicolumn{2}{|c|}{$\begin{array}{c}\text { Estatal } \\
\mathrm{N}=\end{array}$} & \multirow[t]{2}{*}{$\mathrm{Z}$} & \multirow[t]{2}{*}{$d$} & \multirow[t]{2}{*}{ Poder } \\
\hline & $\mathrm{M}$ & O.E. & $\mathbf{M}$ & O.E. & & & \\
\hline & 2.55 & 0.43 & 2.38 & 0.43 & $6.78 * * *$ & 0.40 & 0.99 \\
\hline $\begin{array}{l}\text { Codificación de la } \\
\text { Información }\end{array}$ & 2.38 & 0.41 & 2.27 & 0.39 & $4.69 * * *$ & 0.28 & 0.94 \\
\hline $\begin{array}{l}\text { Recuperación de la } \\
\text { información }\end{array}$ & 2.75 & 0.46 & 2.69 & 0.50 & $2.23 *$ & 0.13 & 0.61 \\
\hline $\begin{array}{l}\text { Apoyo } \\
\text { Al procesamiento }\end{array}$ & 2.73 & 0.46 & 2.64 & 0.48 & $3.44 * *$ & 0.19 & 0.76 \\
\hline $\begin{array}{l}\text { Reflexión Activa } \\
\text { Rendimiento }\end{array}$ & 25.20 & 3.86 & 23.51 & 3.88 & $7.51 * * *$ & 0.44 & 0.99 \\
\hline Escolar & 12.50 & 1.58 & 12.62 & 1.60 & -1.21 & 0.07 & 0.23 \\
\hline
\end{tabular}

Respecto a la tercera hipótesis teórica, en la cual se planta que existen diferencias en las estrategias de aprendizaje, la reflexión activa y el rendimiento escolar según el género de los alumnos de quinto año de secundaria. Fue realizado a través de la prueba $\mathrm{Z}$ de diferencia de medias, en la Tabla 11 se puede observar que existen diferencias estadísticas significativas a favor de los colegios particulares en, adquisición de la información $(\mathrm{Z}=-$ $8.28 \mathrm{P}<.001)$, codificación de la información $(\mathrm{Z}=-5.77 \mathrm{P}<.001)$, recuperación de la información $(\mathrm{Z}=-8: 71 \mathrm{P}<.001)$, apoyo al procesamiento $(\mathrm{Z}=-11.06 \mathrm{P}<.001) \mathrm{Y}$ la reflexión activa $(\mathrm{Z}=-8.42 \mathrm{P}<.001)$.

El análisis del tamaño del efecto, permitió apreciar que las diferencias en los tipos de gestión de los colegio alcanzaron valores medios en el apoyo al procesamiento de la información $(\mathrm{d}=0.64)$, la recuperación de la información $(\mathrm{d}=0.51)$, la reflexión activa $(\mathrm{d}=$ $0.49)$, Y en la adquisición de la información $(\mathrm{d}=0.47)$, mientras que presentan valores pequeños en la codificación de la información $(\mathrm{d}=0.33)$ ), lo cual implica que Ias diferencias son significativas y adecuados en el estudio. En tanto que en el análisis del poder estadístico se apreció que la reflexión activa, la adquisición de la información, la codificación de la información, el apoyo al procesamiento y la recuperación de la información se alcanzó un valor de 0.99. De la revisión de los hallazgos encontrados se puede concluir que la tercera hipótesis es válida. 
Tabla No. 11.- Prueba $Z$ de las escala del ACRA según sexo

\begin{tabular}{|c|c|c|c|c|c|c|c|}
\hline Valores & \multicolumn{2}{|c|}{$\begin{array}{l}\text { Varón } \\
\mathrm{n}=576\end{array}$} & \multicolumn{2}{|c|}{$\begin{array}{l}\text { Mujer } \\
n=624\end{array}$} & $\mathrm{Z}$ & $d$ & Poder \\
\hline $\begin{array}{l}\text { Adquisición de } \\
\text { información }\end{array}$ & $\begin{array}{c}\mathrm{M} \\
2.35\end{array}$ & $\begin{array}{l}\text { D.E. } \\
0.44\end{array}$ & $\begin{array}{r}\mathrm{M} \\
2.55\end{array}$ & $\begin{array}{l}\text { D.E. } \\
0.41\end{array}$ & $-8.28 * * *$ & 0.47 & 0.99 \\
\hline Codificación de & 2.25 & 0.41 & 2.38 & 0.38 & $-5.77 * * *$ & 0.33 & 0.99 \\
\hline $\begin{array}{l}\text { Información } \\
\text { Recuperación }\end{array}$ & 2.59 & 0.47 & 2.83 & 0.47 & $-8.71 * * *$ & 0.51 & 0.99 \\
\hline $\begin{array}{l}\text { información } \\
\text { Apoyo al } \\
\text { procesamiento }\end{array}$ & 2.53 & 0.46 & 2.82 & 0.44 & - & 0.64 & 0.99 \\
\hline Reflexión & 23.32 & 4.03 & 25.19 & 3.67 & $-8.42 * * *$ & 0.49 & 0.99 \\
\hline $\begin{array}{l}\text { Rendimiento } \\
\text { Escol }\end{array}$ & 12.49 & 1.47 & 12.63 & 1.69 & -1.53 & 0.09 & 0.48 \\
\hline
\end{tabular}

$* p<.0 \leqslant * * p<.01 * * * * p<.001$. 


\section{DISCUSIÓN}

El análisis de la escala de reflexión activa, indica que la prueba presenta validez de constructo y confiabilidad por consistencia interna, con lo cual se cumplen con los requerimientos psicométricos básicos para este tipo de instrumentos (Martínez Arias, 1985; Muñiz, 1996; Anastasi y Urbina, 1998) ${ }^{54.55 .56}$. Por otro lado la revisión de los análisis de la escala de estrategias de Aprendizaje (ACRA), permite notar que los resultados psicométricos del análisis de ítemes en las áreas de estrategias, adquisición de información codificación de la información, recuperación de información y apoyo al procesamiento, alcanzan valores significativos de manera que se puede concluir que todos los ítemes son relevantes de conformar el instrumento y además permiten obtener puntajes confiables, estos hallazgos coinciden con los resultados obtenidos por Román y Gallego (1994) ${ }^{9}$, Cano $(1996)^{40}$ y Castro $(2004)^{57}$. Respecto al análisis de la validez de constructo, se aprecia que las áreas del ACRA presentan una sola dimensión latente tal como propone la teoría de las estrategias de aprendizaje y lo demuestran los estudios de estudios Cano (1996) ${ }^{40}$ y Castro $(2004)^{57}$, por lo que se concluye que el instrumento presenta validez.

Los análisis de la bondad de ajuste a la curva normal realizados a través de la prueba de Kolmogorov-Smirnov (Siegel y Castellan, 1995) ${ }^{49}$ permitieron concluir que los puntajes presentan una tendencia a parecerse a la distribución normal, es decir que las estrategias de aprendizaje, la reflexión activa y las notas del rendimiento escolar presentaron una adecuada distribución de manera que fue factible aplicar los análisis y alcanzar resultados relevantes.

La revisión de los niveles alcanzados por los alumnos en las estrategias de aprendizaje, permitió observar que ellos utilizan más las estrategias de recuperación de la información seguidos por las estrategias de apoyo al procesamiento. Estos resultados coinciden con lo reportado por Román y Gallego (1994) y Cano $(1996)^{40}$, de manera que es factible afirmar que los estudiantes de quinto de secundaria evaluados en el presente estudio, al utilizar más las estrategias de autoconocimiento y automanejo, estarían desarrollando sus estrategias metacognitivas de aprendizaje, las cuales van servir de apoyo en el uso de estrategias cognitivas de aprendizaje, de Tal manera que pueden lograr un adecuado procesamiento de la información, lo que les va a permitir ser más independiente en su proceso de aprendizaje. Aunque es necesario destacar que un resultado contrario fue reportado por Castro (2004) ${ }^{57}$ quien encontró que en los colegios estatales predominaron las estrategias de adquisición y codificación, lo cual no favorecería el alcanzar un rendimiento académico óptimo de dichos alumnos.

El análisis del efecto de la reflexión activa y las estrategias de aprendizaje sobre el rendimiento escolar, realizado a través del método multivariado de ecuaciones estructurales, permitió demostrar que el desempeño escolar en los alumnos de quinto de secundario, es afectado de forma positiva y sistemática por la presencia de variables metacognitivas, lo que concuerda con los hallazgos de Page $(1990)^{31}$, Cuevas Renaud y Aguilar Villalobos $(1999)^{32}$ y por Arrieta $(2000)^{33}$ quienes establecen la existencia de modelos teóricos concretos que permiten explicar el rendimiento académico de los alumnos.

Parte de estos hallazgos están en concordancia con lo reportado por Castro (2004) ${ }^{57}$, quien encuentra en su estudio en colegios estatales que las escalas de estrategia de aprendizaje (ACRA) se asocian positivamente con el rendimiento escolar en matemáticas lo cual es interpretado como una evidencia que existe una asociación positiva entre los procesos matacognitivos y el rendimiento académico en las áreas de matemáticas

En relación a las diferencias por el tipo de gestión del colegio Los resultados demostraron que los alumnos provenientes de colegios No estatales alcanzaban mejore' resultados en el uso de la reflexión activa y en el uso de las estrategias de aprendizaje, lo cual puede deberse a la influencia de los aspectos asociados a las diferentes procedimientos 
de enseñanza implementados por los docentes en los centros educativos y que pueden influir en el uso de ciertas estrategias de aprendizaje en sus alumnos.

Respecto a la comparación por sexo, se pudo observar que las mujeres exhibieron un mejor desempeño en el uso de la reflexión activa y la aplicación de las estrategias de aprendizaje, hallazgo que es coincidente por los resultados presentados en los colegios estatales por Castro (2004)57 y que nos indicaría del efecto diferencial que existe en la estimulación que reciben en su contexto educativo.

Los resultados encontrados nos llevan a pensar que tal como 16 indican Nickerson, Perkins y Smith (1994) ${ }^{58}$ las habilidades metacognitivas que implican el manejo de nuestros propios recursos cognitivos y el control de nuestro propio desempeño cognitivo dan también la impresión de ser idóneas para constituir objetivos de entrenamiento de los esfuerzos para mejorar el pensamiento, lo cual conlleva a la necesidad que los alumnos hagan uso de las estrategias metacognitivas para el aprendizaje, pues en las medida que dichas facilitan un aprendizaje autónomo que exige monitoreo y regulación activa de los procesos cognitivos esenciales para planear, ejecutar y resolver problemas, se van a fortalecer los elementos que sirven de base para la consolidación de un aprendizaje efectivo. Finalmente se considera que los hallazgos del presente estudio, han permitido establecer que existe una relación positiva entre la reflexión activa, los procesos metacognitivos y el rendimiento académico en los alumnos de quinto grado de secundaria. de lima, lo cual nos lleva a ampliar nuestra perspectiva acerca de que nuevos caminos se pueden seguir para lograr un real y auténtico incremento de la calidad educativa y con ello tratar de lograr la necesaria excelencia académica en. nuestras escuelas. 


\section{CONCLUSIONES}

Los resultados de la investigación indican que:

1. La Escala de estrategias de aprendizaje (ACRA) presenta confiabilidad por consistencia interna.

2. La Escala de estrategias de aprendizaje (ACRA) presenta validez de constructo.

3. La escala de Reflexión Activa presenta confiabilidad por consistencia interna.

4. La escala de Reflexión Activa presenta validez de constructo.

5. Los puntajes de los alumnos en las áreas de la escala ACRA, la escala de reflexión activa y el rendimiento escolar, presentan distribuciones que se aproximan a la curva normal.

6. Las Estrategias de Aprendizaje y la Reflexión Activa influyen de forma positiva sobre el rendimiento Escolar.

7. Los alumnos procedentes de colegios No estatales presentan mejores Estrategias de aprendizaje que los alumnos procedentes de colegios Estatales.

8. Los alumnos procedentes de colegios No Estatales presentan un mayor desarrollo de la Reflexión Activa que los alumnos procedentes de colegios Estatales.

9. Las mujeres presentan mejores Estrategias de aprendizaje que los varones.

10. Las mujeres presenta un mayor desarrollo de la Reflexión Activa que los varones 


\section{REFERENCIAS}

1. Klauer K. J. Teaching for learning to learn. A critical appraisal with some proposals. Instructional Science, 1988; 17: 351-367.

2. Weinstein e E. \& Mayer, R. E. The teaching of learning strategies. En: Wittrock M.e, Editors. Handbook of research on teaching, $3^{\mathrm{a}}$ ed. Nueva York: MacMillan Publishing Company, 1986.

3. Monereo e Estrategias de Aprendizaje. Madrid: Visor Dis. S. A., 2000.

4. Genovard e \& Gotzens e Psicología de la instrucción. Variable y procesos básicos. Madrid: Síntesis, 1996.

5. Beltrán J.Estrategias de aprendizaje. En: Beltrán J. y Genovard e Editores.

Psicología de la instrucción. Variable y procesos básicos. Madrid. Síntesis, 1996.

6. Borkowsky j. Carr M. \& Pressiey, M.

Spontaneous Strategy Use: Perspectives from Metacognitive Theory. Intelligence, 1987: 11,61-75.

7. Kirby J.R. Cognitive strategies and educational performance. Orlando: Academic Press, 1997.

8. Kurtz-Costes B. E. \& Schneider W. Selfconcept, attributional beliefs, and school achievement: a longitudinal analysis. Contemporary Educational Psychology, 1994; 19, 199-216.

9. Román j. M. Y Gallego S. Escalas de Estrategias de Aprendizaje. ACRA. Madrid: TEA. 1994.

10. Biggs J. B. What do inventories of students' I earn ing processes really measure? A theoretical review and clarification. British ]ournal of Educational Psychology. 1993; 63, 3-19.

11. Yussen S. El rol de la metacognición en las teorías contemporáneas del desarrollo cognitivo. En: Forrest-Presley D.L., MacKinnon G.E., Gary Weller, F. Editors. Metacognición, cognición y performance humana. Orlando, Academic Press. 1985.

12. Boud D., Keogh R. \& Walker, D. Reflection: turning experience into learning. London: Kogan Page. 1988.

13. De La Harpe \&, Radloff A. Do first students reflect on their learning? Why they should and how they can. The Higher Education and Development Society of Australasia (HERDSA), Annual international conference, Auckland. 1998.

14. Mathison S. Critical reflection on classroom practice: Teaching as an investigative activity. In Ross E. W. Editor., Reflective practice in social studies. Washington D. C: NCSC. 1994. 59-68.

15. Biggs. B. \& Moore P. J. The process of learning. Sydney: Prentice Hall. 1993.

16. Angelo T. A. Ten easy pieces: Assessing higher learning in four dimensions. New Directions for Teaching and Learning. 1991; 46.17-31.

17. Ewell P. T. Organizing for learning: A point of entry. Draft prepared for discussion at the 1997 AAHE Summer Academy at Snowbird. National Center for H igher Ed ucation Management Systems (NCHEMS) 1997.

18. Schunk D. H. Goal setting and self efficacy during self-regulated learning. Educational Psychologist. 1990; 25(1), 71-86.

19. Karniol R., \& Ross M. The motivational impact of temporal focus: thinking about the future and the pasto Annual Review of Psychology. 1996; 47, 593-620.

20. Patalano A. L. \& Seifert, C. M.

Oportunistic planning: being reminded of pending goals. Cognitive Psychology. 1997; 34, 1-36.

21. VermuntJ. D. Pracess-oriented instruction in learning and thinking strategies. European Journal of Psychology of Education. 1995; X (4). Pp. 325-349.

22. Justicia F. Metacognición y currículum.

En: Beltrán J. y Genovard C. Editores. Psicología de la instructión 1. Variables y procesos básicos. Madrid: Síntesis, 1996, p 359-381.

23. Zimmerman B. J., Bandura A. \& Martínez Pons, M. Self-motivation for academic attainment: The rale of self efficacy beliefs and personal goal setting. American 
Educational Research Journal, 1992; 29, 663-676.

24. Richardson J. T. E. Using questionnaries to evaluate student learning. In G. Gibbs (Org.), Improving student larning through assesment and evaluation. Oxford: Oxford Centre for Staff Development, 1995.

25. Gibbs G. Improving the quality of student learning. Bristol: technical and Educational Services, 1992.

26. Sobral D. T. Desenvolvimiento e validacao de Escala de Reflexao na Aprendizagem. Psicología: Teoria e Pesquisa.1998; 4(2), Maio -Ago, 173177.

27. Eisler S. H. Adaptación del Inventario de Estilos de Aprendizaje de Kolb. Facultad de Humanidades. PUC, 1989.

28. Escurra M. L. M. Adaptación del Inventario de Estilos de Aprendizaje de Kolb en estudiantes de Psicología pertenecientes diferentes Universidades de Lima Metropolitana. Facultad de Psicología. Tesis para optar el título de Psicólogo. UNMSM, 1991.

29. Hammond M.\& Collins R. Self-directed learning. Critical practice. London: Kogan Page, 1991.

30. Grows D.A. Handbook of research on mathematics teaching and learning. New York. MacMillam Press. 1992.

31. Page M A. Hacia un modelo causal del rendimiento académico. Madrid: C1DEMEC. 1990.

32. Cuevas Renaud y Aguilar Villalobos Metodología para la construcción de modelos estructurales lineales. Rev. Méxicana de psicología, 1999. Vol. 16: 2. 237-243.

33. Arrieta M. Modelo causal de rendimiento y el rendimiento en general académico, diferencias entre el rendimiento en matemáticas. Educación Matemática 2000. Vol. 12; 2 . Agosto 49-61.

34. Aliaga T. J., Ponce D. c., Gutiérrez O.

V., Díaz A. G., Reyes T. Y. Y Pinto L. A. Variables Psicológicas relacionadas con el rendimiento Académico en matemática y estadística en alumnos del primer y segundo año de la facultad de psicología de la UNMSM. Revista de investigación en Psicología UNMSM IPPSI, 2001; Vol. 4 (1), 35-52.

35. García A L., Orellana M. O. \& Canales Q.1. Factores asociados al rendimiento académico en estudiantes de psicología de la UNMSM. Revista de investigación en Psicología - UNMSM-IPPSI, 2002; Vol. 5 (1), 35-52.

36. Aliaga T. J y Giove P. A. Diferencias en el MSLQ y el LASSI entre alumnos del quinto año de secundaria con autoevaluación alta y baja del rendimiento académico en matemática. Revista de Psicología, 2003; Vol. 6 (2), Noviembre, 223-236.

37. Sánchez C. H. Y Reyes M. C.

Metodología y Diseños en la Investigación Científica: Aplicadas a la Psicología, Educación y Ciencias Sociales. Lima: URP. 2002

38. Escurra M. L. M. Adaptación del inventario de estilos de Aprendizaje de Kolb. Revista de Psicología, 1992; X, Vol. X, 1er. y 2do. semestre, 125-142.

39. Escurra M. L. M. La Reflexión en el aprendizaje de los estudiantes del quinto de secundaria de Lima Metropolitana. Revista de Investigación en Psicología. UNMSM-IIPSI, 2002; Vol. 5(2), 55-69.

40. Cano C. E. V. Estrategias metacognitivas y cognitivas en el aprendizaje: estudio en alumnos de quinto de secundaria de NSE alto y medio alto en Lima Metropolitana. Escuela de Graduados. Tesis para optar el grado académico de Magíster. PUCP, 1996.

41. Marín G. Consideraciones metodológicas básicas para conducir investigaciones Psicológicas en América Latina. Acta Psiquiátrica y Psicológica de América de Latina, 1986; Vol. 32, 183-192.

42. Arbuckle J. Introduction to Structural Equation Modeling using AMOS. Austin: Academic Corporation and instructional Technology Services, 2000.

43. Arbuckle, J. AMOS 5.0: Programming reference guide. Chicago: Small Waters Corporation, 2004.

44. Joreskog K. \& Sorbom D. L1SREL 8:

Structural equation Modeling with the SIMPLIS command Language. New Jersey: Lawrence Erlbaum Associates Publishers, 2004. 
45. Borenstein M., Rothstein H. \& Jacob Cohen J. Power and precision: A computer program for statistical power analysis and confidence interval. New Jersey: Biostat. 2004.

46. Kline P. The Handbook of Psychological Testing. London: Routledge, 1995

47. Byrne B. M. A primer of L1SREL: Basic applications and programming for Confirmatory Analytic Models. New York: Springer-Verlag Inc. 1989.

48. Byrne B. M. Structural equation Modeling with L1SREL, PRELIS, and SIMPLIS: Basic concepts, applications and programming. New Jersey: Lawrence Erlbaum Associates Publishers. 1998.

49. Siegel, S. y Castellan N. Estadística no Paramétrica: Aplicada a las ciencias de la conducta. México: Editorial Trillas.1995.

50. Cohen J. Statistical Power analysis for the behavioral sciences. Hillsdale, NJ: Erlbaum. 1988.

51. Cohen J. A Power Prime. Psychological Bulletin.1992. Vol112 (1) Pp. 155-159, 1992

52. Aron A. \& Aron E. Etadística para Psicología. Buenos Aires: Pearson Education. 2001.

53. Clark D. C. Investigación Cuantitativa en Psicología: del diseño experimental al reporte de investigación. México:

Oxford. 2002.

54. Martínez Arias, R. Psicometría: teoría de los test psicológicos y educativos. Madrid: Síntesis, 1985.

55.Muñiz, J. Psicometría. Madrid: Ed. Universitas S. A. 1996

56. Anastasi A. y Urbina S. Test Psicológicos.

México: Editorial Prentice Hall, 1998

57. Castro C. E. A. Estilos de aprendizaje, procesos metacognitivos y rendimiento académico en alumnos de quinto grado de secundaria de centros educativos estatales. Tesis para optar el grado académico de maestra con mención en problemas de aprendizaje. Lima. URP. 2004.

58. Nickerson R., Perkins D. \& Smith E. Enseñar a pensar. Madrid: Ediciones Paidós, 1994. 